\title{
Lesiones de causa externa en menores y mayores de 18 años en un hospital colombiano
}

\author{
Mónica Bejarano ${ }^{1}$ y Luis Fernando Rendón ${ }^{1}$
}

Forma de citar

Bejarano M, Rendón LF. Lesiones de causa externa en menores y mayores de 18 años en un hospital colombiano. Rev Panam Salud Publica. 2009;25(3):234-41.

RESUMEN Objetivos. Definir y comparar las características de las lesiones de causa externa entre los pacientes menores y mayores de 18 años que consultaron al Hospital Mario Correa Rengifo. Métodos. Se realizó un análisis de la información recabada mediante el Sistema de Vigilancia de Lesiones de Causa Externa del hospital Mario Correa Rengifo de Cali, Colombia, comparando menores y mayores de edad, entre enero de 2004 y diciembre de 2007.

Resultados. Se atendieron 4507 menores de 18 años, la mayoría varones (69,3\%), con $75,5 \%$ de las lesiones no intencionales (aunque $88 \%$ de las lesiones intencionales fueron interpersonales y $12 \%$ autoinfligidas). Las lesiones se presentaron con mayor frecuencia en la residencia $(44,1 \%)$ y la calle $(39,5 \%)$; la actividad más frecuente fue la recreación (53,3\%). Los menores de 18 años tuvieron más lesiones en el cráneo (razón de posibilidades [OR] =1,62; $\mathrm{P}=0,0000)$ y la región maxilofacial $(\mathrm{OR}=1,49 ; \mathrm{P}=0,0000)$ que los mayores. En 5,8\% hubo consumo de alcohol y en 2,6\% de drogas, lo que incrementó la ocurrencia de lesiones intencionales (alcohol $\mathrm{OR}=4,25$ y drogas $O R=1,56$ ).

Conclusiones. El número de pacientes menores de 18 años de edad con lesiones de causa externa que son atendidos en el hospital Mario Correa Rengifo aumenta cada vez más. El comportamiento de las lesiones en la niñez y adolescencia es diferente al de los mayores, pues tienen más lesiones no intencionales, que ocurren en el lugar de residencia o en las calles, donde habitualmente juegan. Es importante implementar diversas estrategias, educativas y estructurales para la prevención de este tipo de lesiones.

Palabras clave Violencia, lesiones, salud del niño, salud del adolescente, Colombia.

Los traumatismos son la primera causa de muerte de la población pediátrica en varios países, incluso en los Estados Unidos de América, y causan más defunciones en los niños que todas las otras enfermedades juntas (1-3). La atención médica de las lesiones de causa externa (LCE) requiere un conocimiento especializado, manejo preciso y una observación detallada, de tal forma que el

\footnotetext{
1 Hospital Mario Correa Rengifo, Cali, Colombia. La correspondencia se debe dirigir a Mónica Bejarano, Hospital Mario Correa Rengifo, Carrera 59 No. 11B - 56. Cali, Colombia. Correo electrónico: monicirugia@gmail.com
}

personal de salud pueda reconocer las consideraciones y características especiales y las necesidades únicas del niño traumatizado (4).

La violencia se define como "todas las formas de maltrato físico y emocional, el abuso sexual, el abandono, la negligencia en el cuidado, la explotación comercial o de otro tipo, que resulten en daños reales o potenciales para la salud, supervivencia, desarrollo y dignidad del niño, dentro del contexto de una relación de responsabilidad, confianza o poder" (5). La OMS estima que 40 millones de niños sufren violencia en el mundo y se ha de- mostrado que la mayoría de los menores sometidos a castigos corporales tienen entre 2 y 7 años de edad $(6,7)$. La Organización Mundial de la Salud (OMS) (8) ha exhortado a los Ministerios de Salud para que definan proyectos, programas y cuando sea necesario, cambios administrativos para que su rol en la prevención de LCE sea cada vez más protagónico.

La violencia es el principal problema social y de salud pública en Colombia, de magnitud creciente, que genera grandes costos sociales, políticos, económicos culturales y ambientales (9-12). La alta carga de pacientes con lesiones de causa 
externa que consultan a los servicios de urgencia de los hospitales de Cali, Colombia, es expresión clara de que estas son un problema de salud pública, igual como ocurre en otros lugares del mundo, y no solo una demanda que los servicios de salud deben atender $(3,11-16)$.

$\mathrm{Si}$ bien se sabe que para los diferentes grupos de edad o sexo hay diferentes exposiciones y riesgos de sufrir LCE, como ocurre con las mujeres que tienen más riesgo de sufrir violencia por parte de sus parejas, los niños que sufren lesiones de origen no intencional por caídas y los hombres jóvenes que son mas afectados por violencia interpersonal fuera del hogar (5, 8, 10, 12, 17-19), la información disponible hasta ahora no ha permitido análisis más precisos, de tal manera que se pueda caracterizar con mayor especificidad las diferencias que existen entre grupos de edad y sexo.

Con el fin de superar el vacío de información, desde el año 2003 y como parte de una iniciativa de la OPS y los Centros para el Control y la Prevención de Enfermedades de los Estados Unidos (CDC) $(8,10,17)$, se han desarrollado e implementado en varias instituciones de Colombia sistemas de vigilancia epidemiológica de las lesiones que recogen las variables pertinentes sobre los hechos en que ocurren las LCE (7). El Hospital Mario Correa Rengifo (HMCR) forma parte de ese grupo de hospitales en los que este sistema se ha establecido y funciona hoy en día como parte de la rutina de trabajo en el servicio de urgencias (20). Los resultados de esta estrategia han permitido obtener datos y avanzar en el análisis de las características de los eventos de LCE.

El objetivo del presente estudio es definir y comparar las características de las lesiones de causa externa entre los pacientes menores y mayores de 18 años que fueron atendidos en el HMCR, identificando los factores asociados y otras características que permitan planificar estrategias de prevención de las lesiones en los menores de edad.

\section{MATERIALES Y MÉTODOS}

El Instituto de Investigaciones y Desarrollo en Prevención de Violencia y Promoción de la Convivencia Social (CISALVA) de la Universidad del Valle inició en el año 2003 un proyecto para implementar el Sistema de Vigilancia de Lesiones de Causa Externa (SVLCE) en la ciudad de Cali, Colombia. El CISALVA proporcionó asesoría directa y la OPS y los CDC orientaron y dieron los insumos técnicos requeridos.

Después de que se realizó la prueba piloto en otras instituciones de salud, a finales de 2003 se inició el proceso en el Hospital Mario Correa Rengifo, un hospital público de nivel II de atención en salud. Este hospital tiene una población de referencia de cerca de 250000 personas, donde se realizan 25000 consultas médicas de urgencia y más de 40000 consultas ambulatorias por año; cuenta con cerca de 90 camas de hospitalización y un promedio de 4800 egresos al año. Se localiza en un área de ladera al sur occidente de la ciudad de Cali, Colombia, dentro de una zona de alta vulnerabilidad social, donde los índices de violencia interpersonal están entre los más altos de la ciudad (13).

Se comenzó la capacitación del personal asistencial del Servicio de Urgencias en temas relacionados con vigilancia epidemiológica y violencia. Se adoptó un formulario de la historia clínica diseñado en CISALVA, para ser llenado por el médico al momento de la consulta en el servicio de urgencias, donde parte de la información obtenida depende de los datos suministrados por el paciente o su acompañante. El formulario incluye variables sociodemográficas como edad, género, ocupación, escolaridad y lugar de residencia; variables del evento como intencionalidad o no de la lesión (según el interrogatorio y los hallazgos clínicos), fecha, hora, lugar, actividad y mecanismo; información sobre consumo de alcohol y drogas (como marihuana, bazuco o cocaína) previo al incidente, según el reporte del paciente o su acompañante y la observación clínica del médico; hallazgos clínicos como el sitio anatómico o la naturaleza de la lesión y diagnóstico mediante la décima revisión de la Clasificación Internacional de Enfermedades (CIE-10); severidad de la lesión, determinada mediante índices de trauma como el Índice de trauma revisado (Revised trauma score o RTS) y el Índice de trauma pediátrico (Pediatric trauma score o PTS) (21-24) y destino del paciente o lugar a donde se dirige después de su egreso del hospital.

Por tratarse de un sistema de vigilancia epidemiológica y para facilitar el reporte de información como el consumo de bebidas alcohólicas $\mathrm{u}$ otras sustancias ilícitas, en el interrogatorio solo se tra- taba de obtener una respuesta afirmativa o negativa, sin precisar la dosis ni el tiempo transcurrido entre el consumo y la lesión, de acuerdo con las recomendaciones de Buehler (25).

Cuando el paciente era dado de alta del hospital, la historia clínica se llevaba al archivo, donde una auxiliar de estadística revisaba diariamente los formularios para verificar el registro de los datos; en caso de encontrar omisiones, los registros eran devueltos al médico Coordinador de Urgencias, quien era el responsable de complementar o corregir la información junto con el médico tratante. Luego se procedía a la limpieza de los registros, tratando de corregir errores de transcripción y digitación a una base de datos creada en EPI Info 2003.

Siguiendo la definición contenida en el Artículo 1 de la Convención sobre los Derechos del Niño, modificada en el año 2002 (26), para este estudio se consideró a los "menores de edad" como las personas menores de 18 años, que además es la edad legalmente definida en Colombia para adquirir los derechos y deberes ciudadanos, y a los "mayores de edad" como aquellas personas con 18 años o más de edad. Dado que los tipos de lesiones son frecuentemente un reflejo de la edad del menor (24), para poder analizar aún más las diferencias entre estos grupos, en el estudio los menores de edad se dividieron en cuatro grupos según los ciclos vitales o etapas del desarrollo: menores de 1 año, preescolares (entre 1 y 5 años), escolares (entre 6 y 12 años) y adolescentes (entre 13 y 17 años).

Se evaluaron las variables teniendo en cuenta la gravedad de las lesiones (medida por los índices de trauma) y la intencionalidad de las lesiones según la recomendación de la OMS en el Informe mundial sobre la violencia y la salud (8), donde se categorizaron las lesiones en no intencionales (anteriormente llamados accidentes) e intencionales (subdividida en violencia interpersonal y autoinflingida). Para las lesiones de tránsito, se analizó además el tipo de transporte y de uso (por ejemplo, peatón, conductor, pasajero, otro).

Los datos se analizaron en el programa STATA 8.2. Se calcularon frecuencias, porcentajes, medidas de asociación como razón de posibilidades (OR, por su sigla en inglés), intervalos de confianza, pruebas de significancia estadística $\left(X^{2} \mathrm{y}\right.$ prueba exacta de Fisher) y valor de $P$. 


\section{RESULTADOS}

Entre enero de 2004 y diciembre de 2007 hubo un aumento progresivo en las consultas en ambos grupos, sobre todo en los pacientes menores de edad; se registraron 12148 pacientes con lesiones de causa externa, de los cuales 4507 $(37,1 \%)$ fueron menores de 18 años. La ocurrencia de las lesiones según el día de la semana se presenta de manera diferencial: en los adultos las lesiones predominan los fines de semana, mientras que en los menores de edad la distribución de la ocurrencia de las lesiones es muy similar entre los diferentes días de la semana y oscila desde $12,7 \%$ los martes hasta $18,5 \%$ los domingos.

La mediana de edad de todos los pacientes atendidos fue de 23 años, con un rango intercuartil (RI) comprendido entre 13 y 38 años. Dentro de la población de menores de edad, la mediana correspondió a 10 años (RI de 5 a 15 años). Se atendieron 63 niños menores de un año, $1330(29,5 \%)$ en edad preescolar, $1504(33,4 \%)$ en edad escolar y 1605 $(35,6 \%)$ adolescentes.

Con relación a la escolaridad, la población atendida reportó contar con estudios primarios básicos $(35,4 \%)$ y secundarios $(52,1 \%)$ en su mayoría; sin embargo, $3 \%$ (105 menores y 267 adultos) no tenían escolaridad alguna.

Los pacientes provienen en su mayoría de las comunas $18(62,1 \%)$ y $20(6,1 \%)$, que corresponden a la zona de ladera de la ciudad, y 10,4\% provienen de la zona oriental (comunas 13, 14, 15 y 16) denominada "distrito de Agua Blanca". Ambas áreas se caracterizan por sus condiciones socioeconómicas pobres. La georreferenciación por barrio también permite identificar zonas de focalización de los pacientes, como es el caso de los ba- rrios Los Chorros, Alto Nápoles y Prados del Sur, conocidos en la región por su alto índice de violencia e inseguridad (13).

Las lesiones intencionales representaron $24,5 \%$ del total de los casos atendidos. Para los menores de edad la proporción fue de $12,7 \%$, en contraste con $31,5 \%$ de los adultos. Esta diferencia presentó significación estadística $\left(X^{2}=521,6 P=0,0001\right)$. Dentro del grupo de menores con lesiones intencionales, 67 (12\%) fueron autoinfligidas y $491(88 \%)$, resultado de violencia interpersonal o de agresiones.

En el cuadro 1 se presenta la distribución de los sitios donde suceden las lesiones, que en los menores de edad ocurren con mayor frecuencia en el hogar $(44,1 \%)$, mientras en los mayores acontecen en la calle $(50,0 \%)$. Para los niños menores de 6 años el porcentaje en el hogar es mucho más alto, a diferencia de los adolescentes, entre quienes más de la mitad de las lesiones se presentan en la calle, igual que en los adultos. Respecto al género, se encuentra que en las niñas es mayor el porcentaje de lesiones en el hogar $(55,7 \%)$ que en la calle $(31,7 \%)$, contrario a lo que pasa en los niños que se lesionan más en la calle $(43,4 \%)$ y se asocian más con lesiones intencionales.

Entre las lesiones ocurridas en los menores de edad en la calle o vía pública, se encontró que $38 \%$ fueron en bicicleta y $27 \%$ en motocicleta. Por otro lado, se registró que en el momento de la lesión $29 \%$ eran peatones, $44,3 \%$ conductores y $24,1 \%$ pasajeros.

En el cuadro 2 se observa que más de la mitad de las lesiones en menores de edad $(53,3 \%)$ ocurrieron mientras realizaban actividades recreativas. Llama la atención el porcentaje de adolescentes $(8,5 \%)$ que refirieron el consumo de bebidas alcohólicas cuando sucedió el evento.
En cuanto a los mecanismos de las lesiones, el primer lugar lo ocupan las caídas $(34,9 \%)$, seguidas de las heridas por arma cortopunzante $(22,5 \%)$, cuyo porcentaje incrementa a medida que aumenta el rango de edad en cada grupo (cuadro 3). No existen diferencias cuando se compara por género el mecanismo u objeto de la lesión en los menores de 18 años.

Durante el interrogatorio médico realizado en el servicio de urgencias se determinó que hubo ingesta de alcohol previo al evento en $264(5,8 \%)$ menores; cuando la respuesta fue negativa, se sospechó por la evaluación médica en 52 de estos pacientes $(1,1 \%)$, lo que representa una significación estadística con respecto a los mayores de 18 años, quienes consumen cuatro veces más bebidas alcohólicas $\left(X^{2}=695,07, P=0,000\right)$. El porcentaje fue mayor en los adolescentes $(13,4 \%)$ que en el resto de los niños y niñas $(3,5 \%)$ y no hubo diferencia por género en el consumo por parte de los menores. El porcentaje de los menores de 18 años que refirieron consumo de bebidas alcohólicas fue más alto cuando las lesiones ocurrieron en bares o cantinas $(62,9 \%)$ y en la calle o vía pública $(10,5 \%)$ y cuando el mecanismo fue herida por proyectil de arma de fuego $(23,6 \%)$ y arma cortopunzante $(14,8 \%)$. Respecto a la intencionalidad de las lesiones en los menores de edad, es evidente que el consumo de bebidas alcohólicas juega un papel muy importante (cuadro 4).

En relación con los psicofármacos (comúnmente llamados drogas), 118 (2,6\%) menores informaron haberlos consumido antes de la lesión y mediante la evaluación médica se sospechó en $50(1,1 \%)$ más; en este caso también se encontró una diferencia con significación estadística con respecto a los mayores, que las

CUADRO 1. Lugar de ocurrencia de las lesiones según grupo de edad ( $n=12$ 143), Cali, Colombia, 2004-2007

\begin{tabular}{|c|c|c|c|c|c|c|c|c|c|c|c|c|c|c|}
\hline \multirow[b]{3}{*}{ Lugar } & \multicolumn{10}{|c|}{ Menores de 18 años de edad } & \multirow{2}{*}{\multicolumn{2}{|c|}{$\begin{array}{c}\text { Mayores de } \\
18 \text { años }\end{array}$}} & \multirow{2}{*}{\multicolumn{2}{|c|}{ Total }} \\
\hline & \multicolumn{2}{|c|}{$<1$ año } & \multicolumn{2}{|c|}{$1-5$ años } & \multicolumn{2}{|c|}{$6-12$ años } & \multicolumn{2}{|c|}{ 13-18 años } & \multicolumn{2}{|c|}{ Total } & & & & \\
\hline & No. & $\%$ & No. & $\%$ & No. & $\%$ & No. & $\%$ & No. & $\%$ & No. & $\%$ & No. & $\%$ \\
\hline Casa & 54 & 85,7 & 923 & 69,4 & 616 & 40,9 & 392 & 24,4 & 1985 & 44,1 & 2387 & 31,2 & 4372 & 36,0 \\
\hline Escuela & & & 48 & 3,6 & 149 & 9,9 & 106 & 6,6 & 303 & 6,7 & 40 & 0,5 & 343 & 2,8 \\
\hline Calle & 9 & 14,3 & 307 & 23,1 & 610 & 40,5 & 851 & 53,0 & 1777 & 39,5 & 3821 & 50,0 & 5598 & 46,1 \\
\hline Trabajo & & & 10 & 0,7 & 9 & 0,6 & 72 & 4,5 & 91 & 2,0 & 888 & 11,6 & 979 & 8,0 \\
\hline Bar & & & & & 1 & 0,1 & 26 & 1,6 & 27 & 0,6 & 146 & 1,9 & 173 & 1,4 \\
\hline Otro & & & 38 & 2,8 & 112 & 7,4 & 146 & 9,1 & 296 & 6,6 & 286 & 3,7 & 582 & 4,8 \\
\hline Sin datos & & & 4 & 0,3 & 7 & 0,5 & 12 & 0,7 & 23 & 0,5 & 73 & 0,9 & 96 & 0,8 \\
\hline Total & 63 & 100 & 1330 & 100 & 1504 & 100 & 1605 & 100 & 4502 & 100 & 7641 & 100 & 12143 & 100 \\
\hline
\end{tabular}

Fuente: Sistema de vigilancia de lesiones de causa externa, Hospital Mario Correa Rengifo, Cali, Colombia. 
CUADRO 2. Actividades que realizaban al momento de las lesiones según grupo de edad ( $n=12$ 143), Cali, Colombia, 2004-2007

\begin{tabular}{|c|c|c|c|c|c|c|c|c|c|c|c|c|c|c|}
\hline \multirow[b]{3}{*}{ Actividad } & \multicolumn{10}{|c|}{ Menores de 18 años de edad } & \multirow{2}{*}{\multicolumn{2}{|c|}{$\begin{array}{c}\text { Mayores de } \\
18 \text { años }\end{array}$}} & \multirow{2}{*}{\multicolumn{2}{|c|}{ Total }} \\
\hline & \multicolumn{2}{|c|}{$<1$ año } & \multicolumn{2}{|c|}{$1-5$ años } & \multicolumn{2}{|c|}{ 6-12 años } & \multicolumn{2}{|c|}{ 13-18 años } & \multicolumn{2}{|c|}{ Total } & & & & \\
\hline & No. & $\%$ & No. & $\%$ & No. & $\%$ & No. & $\%$ & No. & $\%$ & No. & $\%$ & No. & $\%$ \\
\hline Trabajo dependiente & & & 5 & 0,4 & 12 & 0,8 & 46 & 2,9 & 63 & 1,4 & 659 & 8,6 & 722 & 5,9 \\
\hline Oficio informal & & & 11 & 0,8 & 18 & 1,2 & 93 & 5,8 & 122 & 2,7 & 918 & 12,0 & 1040 & 8,5 \\
\hline Labores personales & 6 & 9,5 & 142 & 10,7 & 197 & 13,1 & 299 & 18,6 & 644 & 14,3 & 2027 & 26,5 & 2671 & 22,0 \\
\hline Estudiando & & & 14 & 1,0 & 65 & 4,3 & 50 & 3,1 & 129 & 2,8 & 40 & 0,5 & 169 & 1,4 \\
\hline Deportes & & & 30 & 2,2 & 106 & 7,0 & 137 & 8,5 & 273 & 6,0 & 163 & 2,1 & 436 & 3,6 \\
\hline Viajando & 5 & 7,9 & 53 & 4,0 & 85 & 5,6 & 155 & 9,6 & 298 & 6,6 & 785 & 10,3 & 1083 & 8,9 \\
\hline Recreación & 40 & 63,5 & 963 & 72,4 & 896 & 59,6 & 502 & 31,2 & 2401 & 53,3 & 1131 & 14,8 & 3532 & 29,1 \\
\hline Tomando licor & & & 12 & 0,9 & 9 & 0,6 & 136 & 8,5 & 157 & 3,5 & 1130 & 14,8 & 1287 & 10,6 \\
\hline Otra & 8 & 12,7 & 63 & 4,7 & 56 & 3,7 & 99 & 6,2 & 226 & 5,0 & 379 & 4,9 & 605 & 5,0 \\
\hline Sin datos & 4 & 6,3 & 37 & 2,8 & 60 & 4,0 & 88 & 5,5 & 189 & 4,2 & 409 & 5,3 & 598 & 4,9 \\
\hline Total & 63 & 100 & 1330 & 100 & 1504 & 100 & 1605 & 100 & 4502 & 100 & 7641 & 100 & 12143 & 100 \\
\hline
\end{tabular}

Fuente: Sistema de vigilancia de lesiones de causa externa, Hospital Mario Correa Rengifo, Cali, Colombia.

CUADRO 3. Mecanismo u objeto de las lesiones según grupo de edad ( $n=12$ 143), Cali, Colombia, 2004-2007

\begin{tabular}{|c|c|c|c|c|c|c|c|c|c|c|c|c|c|c|}
\hline \multirow{3}{*}{$\begin{array}{c}\text { Mecanismo u } \\
\text { objeto de la lesión }\end{array}$} & \multicolumn{10}{|c|}{ Menores de 18 años de edad } & & & & \\
\hline & \multicolumn{2}{|c|}{$<1$ año } & \multicolumn{2}{|c|}{$1-5$ años } & \multicolumn{2}{|c|}{ 6-12 años } & \multicolumn{2}{|c|}{ 13-18 años } & \multicolumn{2}{|c|}{$\begin{array}{l}\text { Total de } \\
\text { menores }\end{array}$} & \multicolumn{2}{|c|}{$\begin{array}{c}\text { Mayores de } \\
18 \text { años }\end{array}$} & \multicolumn{2}{|c|}{ Total } \\
\hline & No. & $\%$ & No. & $\%$ & No. & $\%$ & No. & $\%$ & No. & $\%$ & No. & $\%$ & No. & $\%$ \\
\hline Caídas & 39 & 57,3 & 762 & 57,3 & 758 & 50,4 & 485 & 30,2 & 2044 & 45,4 & 2198 & 28,7 & 4242 & 34,9 \\
\hline Cortante & 3 & 4,7 & 110 & 8,3 & 181 & 12,0 & 380 & 23,7 & 674 & 15,0 & 2057 & 26,9 & 2731 & 22,5 \\
\hline Contuso & 5 & 7,9 & 153 & 11,5 & 211 & 14,0 & 236 & 14,7 & 605 & 13,4 & 1101 & 14,4 & 1706 & 14,0 \\
\hline Arma de fuego & 1 & 1,6 & 1 & 0,1 & 7 & 0,5 & 122 & 7,6 & 131 & 2,9 & 488 & 6,4 & 619 & 5,1 \\
\hline Transporte & 1 & 1,6 & 83 & 6,2 & 159 & 10,6 & 188 & 11,7 & 431 & 9,6 & 996 & 13,0 & 1427 & 11,7 \\
\hline Cuerpo extraño & 2 & 3,2 & 50 & 3,7 & 23 & 1,5 & 22 & 1,4 & 97 & 2,1 & 181 & 2,4 & 285 & 2,3 \\
\hline Mordedura & 1 & 1,6 & 50 & 3,7 & 61 & 4,0 & 24 & 1,5 & 136 & 3,0 & 96 & 1,2 & 232 & 1,9 \\
\hline Intoxicación & 2 & 3,2 & 35 & 2,6 & 8 & 0,5 & 57 & 3,5 & 102 & 2,2 & 117 & 1,5 & 219 & 1,8 \\
\hline Quemadura & 5 & 7,9 & 18 & 1,3 & 28 & 1,9 & 20 & 1,2 & 71 & 1,6 & 86 & 1,1 & 157 & 1,3 \\
\hline Sexual & & & 2 & 0,1 & 5 & 0,3 & 5 & 0,3 & 12 & 0,2 & 8 & 0,1 & 20 & 0,1 \\
\hline Otros & 4 & 6,3 & 59 & 4,5 & 55 & 3,6 & 55 & 3,4 & 173 & 3,8 & 245 & 3,2 & 418 & 3,4 \\
\hline Sin datos & & & 7 & 0,5 & 8 & 0,5 & 11 & 0,7 & 26 & 0,6 & 61 & 0,8 & 87 & 0,7 \\
\hline Total & 63 & 100 & 1330 & 100 & 1504 & 100 & 1605 & 100 & 4502 & 100 & 7641 & 100 & 12143 & 100 \\
\hline
\end{tabular}

Fuente: Sistema de vigilancia de lesiones de causa externa, Hospital Mario Correa Rengifo, Cali, Colombia.

CUADRO 4. Regiones corporales lesionadas en menores y mayores de edad según grupo de edad ( $n=12$ 148), Cali, Colombia, 2004-2007

\begin{tabular}{lrrrrrr}
\hline & \multicolumn{2}{c}{ Menores de 18 años } & & \multicolumn{2}{c}{ Mayores de 18 años } & \\
\cline { 2 - 3 } Región corporal & \multicolumn{1}{c}{ No. } & $\%$ & & No & $\%$ & OR (IC95\%) ${ }^{\mathrm{a}}$ \\
\hline Cráneo & 1004 & 22,3 & & 1144 & 15,0 & $1,62(1,4797-1,7905)$ \\
Ojos & 120 & 2,6 & & 286 & 3,7 & $0,70(0,5618-0,8767)$ \\
Maxilofacial & 672 & 14,9 & & 802 & 10,5 & $1,49(1,3367-1,6703)$ \\
Cuello & 89 & 2,0 & & 248 & 3,2 & $0,60(0,4647-0,7705)$ \\
Tórax & 345 & 7,6 & & 1293 & 16,9 & $0,40(0,3584-0,4620)$ \\
Abdomen & 182 & 4,0 & & 547 & 7,1 & $0,54(0,4577-0,6503)$ \\
Columna & 64 & 1,4 & & 256 & 3,3 & $0,41(0,3101-0,5500)$ \\
Pelvis & 98 & 2,2 & & 224 & 2,9 & $0,73(0,5728-0,9402)$ \\
Miembro superior & 1602 & 35,5 & & 3102 & 40,6 & $0,80(0,7471-0,8714)$ \\
Miembro inferior & 986 & 21,9 & & 1995 & 26,1 & $0,79(0,7256-0,8655)$ \\
\hline
\end{tabular}

Fuente: Sistema de vigilancia de lesiones de causa externa, Hospital Mario Correa Rengifo, Cali, Colombia.

a Razón de posibilidades (intervalo de confianza al 95\%).

utilizan más $\left(X^{2}=291,23, P=0,000\right)$. Aunque se registró consumo de psicofármacos en niños desde los 2 años de edad (suministrados por uno de los padres), el porcentaje aumenta en los adolescentes $(7,1 \%)$, sin diferencia importante por género. Igual que para el alcohol, el porcentaje de los menores de 18 años que refi- rieron consumo de drogas fue más alto cuando las lesiones ocurrieron en bares o cantinas $(29,6 \%)$ y en la calle o vía publica $(6,4 \%)$, y cuando el mecanismo fue herida 
CUADRO 5. Análisis de asociación para intencionalidad y severidad de las lesiones en menores de edad ( $n=12$ 148), Cali, Colombia, 2004-2007

\begin{tabular}{lccc}
\hline \multicolumn{1}{c}{ Variable } & OR $^{\mathrm{a}}$ & $\mathrm{IC}^{2} \%^{\mathrm{b}}$ & $P$ \\
\hline Sexo masculino & 1,54 & $1,38-1,72$ & 0,000 \\
Consumo de alcohol & 4,25 & $3,77-4,80$ & 0,000 \\
Uso de drogas & 1,56 & $1,27-1,91$ & 0.000 \\
Trauma de cuello & 1,49 & $1,15-1,93$ & 0,002 \\
Trauma de tórax & 3,02 & $2,66-3,44$ & 0,000 \\
Trauma de abdomen & 3,48 & $2,89-4,19$ & 0,000 \\
Contusión & 1,52 & $1,18-1,96$ & 0,001 \\
Herida cortante & 1,73 & $1,53-1,96$ & 0,000 \\
Herida profunda & 11,80 & $10,00-13,91$ & 0,000 \\
\hline Fuente: Sistema de vigilancia de lesiones de causa externa, Hospital Mario Correa Rengifo, \\
Cali, Colombia. \\
a Razón de posibilidades. \\
b Intervalo de confianza al 95\%.
\end{tabular}

por proyectil de arma de fuego $(13,0 \%)$ y arma cortopunzante $(8,1 \%)$. También se observó que el consumo de drogas se asocia con aumento del riesgo intencionalidad de las lesiones en menores de edad (cuadro 4).

La intencionalidad se ha asociado a la gravedad de la lesión y al sitio anatómico afectado en el evento violento (27). Al evaluar las regiones corporales comprometidas (cuadro 5) se encuentra que los menores de edad presentan más lesiones en la cabeza y región maxilofacial, en comparación con los mayores, con una diferencia estadísticamente significante.

El destino luego de la atención inicial de los pacientes se utilizó como un indicador de la gravedad de la lesión; se observó que después de la atención en urgencias son dados de alta con mayor frecuencia los menores de 18 años de edad $(83,1 \%)$ que los mayores $(72,7 \%)$ y que requirieron menos hospitalizaciones ( $8,3 \%$ vs. $15,7 \%$ respectivamente) o remisiones a otras instituciones de salud $(8,5 \%$ vs. $11,8 \%$ ), diferencias con significación estadística.

En relación con la severidad de la lesión (medida por el Índice de trauma pediátrico o PTS en los menores de 18 años) y la intencionalidad (cuadro 4), se encontró que las heridas cortantes profundas y las lesiones en tórax y abdomen tienen una asociación mayor (OR más altos) en los menores, lo que sugiere que, aunque estas lesiones son menos frecuentes en este grupo en comparación con los adultos, cuando suceden tienen una mayor gravedad.

\section{DISCUSIÓN}

En el presente estudio se observa que ha aumentado la tendencia de la consulta de pacientes con lesiones de causa externa al servicio de urgencias del HMCR. El registro mensual de los casos muestra, además de un aumento en dicha tendencia, una estacionalidad marcada con picos de mayor frecuencia hacia la mitad y el fin de cada año. El primer pico coincide con las vacaciones estudiantiles en Colombia y el segundo se relaciona con las fiestas de fin de año, cuando además se realiza la Feria de Cali (17), donde muchas de las actividades recreativas se relacionan con la ingesta de alcohol; esta información sirve para planear la oferta de los servicios de urgencia. Sin embargo, no es posible tener un censo real ni actualizado porque no se sabe exactamente el número de habitantes de esa zona de la ciudad, ya que hay invasiones y migraciones frecuentes por tratarse de un área marginal de ladera (13). Además, como es un hospital perteneciente a la red pública de salud del departamento, atiende pacientes provenientes de toda la ciudad, no solo de su área de influencia. Mayor información sobre los barrios de donde proviene el mayor número de pacientes permitirá a los gobernantes focalizar las intervenciones para obtener mejores resultados (17).

También se puede decir que la gravedad de las lesiones ha aumentado, pues al consultar la información de la mortalidad por violencia en esta comuna se encontró un aumento en el volumen de los años de vida potencial perdidos por causas violentas tanto en menores como en mayores de edad (13). Por otro lado, al interior del hospital y debido al cierre del programa de atención para pacientes de cirugía de urgencias en la segunda mitad del año 2007, se observó una disminución en el porcentaje de lesiones intencionales, con aumento de las no in- tencionales, sobre todo en los menores de edad.

Nuestro hallazgo de que la primera causa de lesiones fueron las caídas y la mayor cantidad de las lesiones ocurren en la casa, sobre todo en los niños y niñas pequeños, coincide con lo publicado por otros autores $(1,3,24,28)$.

Los accidentes de tránsito son la primera causa de muertes violentas en muchos lugares del mundo (2, 3, 18, 29-33), lo cual no se pudo comprobar en nuestro estudio debido a que el hospital no es un centro de referencia para este tipo de pacientes, tanto por su ubicación (zona de ladera) como por su nivel de complejidad. Sin embargo, en el estudio la mayoría de los lesionados en la calle eran peatones o ciclistas, condición que genera una alta carga de morbilidad y discapacidad (2, 3, 33). La georreferenciación permite ubicar los lugares con mayor número de lesionados en las vías públicas, para identificar las áreas prioritarias donde se debe mejorar la infraestructura de las calles y la señalización, además de las campañas de educación enfocadas a los niños y adolescentes $(29,31-33)$.

El Informe mundial sobre la violencia contra los niños confirma que esta ocurre en todas partes del mundo (5). La edad, el género, la densidad poblacional, la pobreza y la inequidad influyen en la violencia mediante mecanismos distintos, pero el consumo de bebidas alcohólicas y drogas aumenta la predisposición a exhibir conductas agresivas y violentas (7, $8,10,11,17,19)$. El alcohol es un factor importante en la violencia contra y por los adolescentes en la comunidad y varias publicaciones han demostrado vínculos entre alcohol y violencia en adolescentes $(5,8,17,28)$, así como entre consumo de alcohol y accidentes de tránsito $(2,18,28,30)$. Además, según Hernández (34) hay un mayor consumo de alcohol como medio de socialización entre adolescentes a pesar de la legislación existente en el país que prohíbe dicho consumo. Los resultados del estudio muestran cómo el consumo de alcohol y drogas tiene una asociación con intencionalidad de la lesión en los menores de edad.

Cuando se monitorizan comportamientos que pueden tener implicaciones legales, el potencial del subregistro puede sobrepasar la conveniencia de las entrevistas; por eso en la vigilancia epidemiológica, ante la necesidad de simplificar para facilitar su amplio uso, las 
definiciones utilizan limitadas opciones de respuestas (como si o no), que pueden ser aplicadas rápidamente en una variedad de escenarios o constancias, mientras los clínicos adicionan a este criterio su entendimiento subjetivo de cada paciente (25). Estas diferencias de perspectiva deben ser tenidas en cuenta porque pueden generar confusión cuando son vistas por el salubrista o por el clínico, e incluso cuando las definiciones establecidas para la vigilancia son utilizadas para otros propósitos.

Quizá por falta de educación a los médicos, el PTS se calculó en un bajo porcentaje $(11 \%)$ de los pacientes menores de edad, pero con los datos obtenidos se observó que el tipo de pacientes que ingresa a nuestra institución en más de 98\% presenta lesiones leves (el puntaje de PTS es mayor de 8 puntos y además rápidamente son dados de alta luego de la atención inicial de urgencia), aunque estos resultados pueden estar influenciados por el nivel de atención de nuestro hospital, que es intermedio, y no es un centro de referencia de trauma (24).

La gravedad de la lesión tiene grandes implicaciones para la atención en los servicios de salud y los factores pronósticos de sus resultados. Un primer abordaje muestra que la alta frecuencia de las lesiones no intencionales congestiona los servicios de salud y genera una alta carga de la enfermedad (discapacidad y costos de atención); por otra parte, la intencionalidad tiene una asociación mayor con la gravedad en los menores sugiriendo nuevamente una mayor vulnerabilidad de esta población.

Los niños y niñas requieren consideraciones especiales cuando se enfoca cualquier tipo de lesión pues son más vulnerables a las fuerzas sobre su cuerpo comparado con los adultos; viven en un mundo diseñado para adultos y con frecuencia son incapaces de juzgar los peligros inherentes en muchas situaciones peligrosas (1). El desarrollo corporal y psicomotor se correlaciona con los mecanismos de las lesiones en los menores de edad. Las lesiones de cabeza, solas o asociadas a otros traumas, son las más frecuentes y severas y causan la mayoría de las muertes $(3-5,8,24)$. Esto se explica porque en los niños el tamaño de la cabeza es proporcionalmente más grande, el centro de gravedad más alto y el sostén cefálico relativamente deficiente, comparado con los adultos, lo que favorece su traumatismo.
El mensaje central del Estudio sobre violencia contra los niños y otras publicaciones es que ninguna violencia contra los niños es justificable y que toda violencia contra los niños es prevenible (5, 9). Como lo manifiesta la OMS, en general, la respuesta del sector salud a la violencia es marcadamente reactiva y terapéutica. Ya que esta respuesta tiende a ser fragmentada en áreas de interés especial y experticia, la visión más amplia $y$ las conexiones entre las diferentes formas de violencia con frecuencia es ignorada $(1,12)$. La violencia, sin embargo, es un fenómeno complejo y necesita ser enfocada de una manera más comprehensiva y holística $(8,35)$.

Por eso la obligación del sector salud no debe limitarse a la atención de los pacientes que acuden a los servicios de urgencias, ni llevar un registro minucioso de los muertos, sino que debe orientar las acciones de promoción de las conductas de vida saludable y de prevención de situaciones o conductas de riesgo, mediante charlas educativas breves en el servicio de urgencias, para evitar la presentación de hechos violentos que pongan en peligro la vida de los ciudadanos (12).

La vigilancia es un proceso continuo y sistemático de recolección, análisis, interpretación y diseminación de información descriptiva para monitorizar problemas de salud. Los sistemas de vigilancia pueden ser descritos como lazos de información, con información que entra en la organización que recolecta e información que es retornada a aquellos que la necesitan (25). La vigilancia en sí no realiza la acción, pero sí plantea las alternativas, controla su dinamismo, revisa la estrategia, plantea la evaluación y los ajustes, las posibles metas (36). Se debe instalar la vigilancia cuando hay esfuerzos encaminados a la acción, de lo contrario hay duda sobre un esfuerzo que no tendrá resultados en el cambio de la tendencia de ese evento. Se deben implementar sistemas de vigilancia para aquellas enfermedades que pueden ser controladas, o que necesitan una acción paliativa cuando conducen a una alta mortalidad (36), como es el caso de la violencia en Colombia.

Los resultados del presente estudio son de mucha utilidad para sensibilizar sobre la necesidad de proponer estrategias de prevención de lesiones en el hogar, que es el lugar donde ocurre la mayor cantidad de lesiones en los menores. El gobierno local podría implemen- tar estrategias que han demostrado buenos resultados, como las escuelas de padres, integrando además a los educadores $(1,3,11,18,35)$ mediante reuniones regulares, sobre todo en los barrios que han mostrado la mayor frecuencia de residentes con lesiones. Por otro lado, si se logra adecuar escenarios apropiados para la práctica de deportes o actividades recreativas se disminuirá el número de menores que juegan en las calles, exponiéndose a ser lesionados, y se utilizará mejor el tiempo libre, que ha demostrado que ayuda a disminuir la probabilidad de que un menor se convierta en agresor (11).

El hecho de que la información se obtenga mediante interrogatorio del paciente o su acompañante se convierte en una limitante pues pueden ocultar deliberadamente datos, especialmente cuando se trata de lesiones intencionales o se ha consumido alcohol o drogas $(19,25,29)$. Otra limitante fue el escaso registro de algunos datos por parte del médico en el formato diseñado, que alcanza niveles críticos como en el caso de los datos requeridos para el cálculo del PTS. Es necesario alcanzar una mayor concientización del personal de salud sobre la importancia de la utilización de los índices de trauma, ampliamente demostrado en la literatura mundial como herramienta para realizar triage, evaluar el pronóstico de un paciente y definir la necesidad de traslado a otro centro de salud especializado en trauma (24). Finalmente, la falta de conocimiento en las instituciones gubernamentales sobre la población de la zona, impide obtener tasas precisas y planear estrategias de una manera más apropiada, lo que constituye una limitante más.

El Sistema de vigilancia de lesiones en el HMCR ha proporcionado una gran información, no solo en la caracterización de la lesiones, sino también en el establecimiento de la asociación entre los factores de riesgo conocidos y la severidad del problema. El personal de salud ha conocido de primera mano la información que recolectan en las historias clínicas de los lesionados; las autoridades administrativas de la institución han reorganizado la prestación de los servicios de urgencias (30) y los equipos interdisciplinarios para la atención de los casos según las necesidades detectadas mediante esta herramienta; las autoridades políticas de la ciudad han observado los buenos resultados del sistema y tienen previsto su implementación en todas las instituciones 
de la ciudad y, finalmente, la comunidad ha aumentado su preocupación por el problema y se espera que en un futuro cercano se trabaje mancomunadamente por políticas de promoción de la salud en torno a la prevención de las lesiones.
Agradecimientos. Los autores expresan su más sentido agradecimiento al personal del Servicio de Urgencias del HMCR por su ardua labor en la atención de los pacientes y el empeño en el diligenciamiento de las historias clínicas y al grupo humano del área de Estadística, por el trabajo de digitación y la conservación de la base de datos. Así mismo a CISALVA por su asesoría para la implementación de este proyecto en el HMCR.

\section{REFERENCIAS}

1. Ameratunga SN, Hyden AA, Sultana S. Setting priorities for child health and research: the neglected burden of injuries. Salud $\mathrm{Pu}-$ blica Mex. 2008;50 supl 1:S115-7.

2. Arias S. Factores de riesgo asociados a accidentes de tránsito en menores de 19 años. Pediatría (Asunción). 2005;32(1). Hallado en: http://www.spp.org.py/revistas/ed_2005/ facriesgo_vol1_2005.htm. Acceso el 8 de junio de 2008.

3. Zayas Mujica R, Cabrera Cárdenas U, Simón Cayón D. ¿Accidentes infantiles o lesiones no intencionales? Rev Cubana Pediatr (Online). 2007;79(1). Hallado en: http://scielo.sld.cu/ scielo.php?script=sci arttext\&pid=S003475312007000100009\&lng=es\&nrm=iso\&tlng= es. Acceso el 8 de junio de 2008.

4. Alterman D, Daley BJ, Kennedy A, Raju R, Lee S. Ph. Considerations in pediatric trauma. E-medicine [Sitio en Internet]. Hallado en: http://www.emedicine.com/med/topic3223 .htm. Acceso el 9 de marzo de 2009.

5. Pinheiro PS. World Report on Violence against Children, United Nations SecretaryGeneral's Study on Violence against Children. New York: United Nations; 2006. Hallado en: http://www.violencestudy.org/ r229. Acceso en septiembre de 2007.

6. Concha-Eastman A, Villaveces A. Guías para el diseño, implementación y evaluación de sistemas de vigilancia epidemiológica de violencia y lesiones. Washington, DC: Organización Panamericana de la Salud; 2001.

7. Buvinic M, Morrison A, Orlando M. Violencia, crimen y desarrollo social en América Latina y el Caribe. Hallado en: http:// www.flacso.org/biblioteca/violencia.doc Acceso en septiembre de 2005.

8. World Health Organization. World report on violence and health. Geneva: WHO; 2002.

9. Colombia. Acuerdo 05 por el cual se desarrolla la estructura interna del Instituto Nacional de Medicina Legal y Ciencias Forenses. Diario Oficial, junio 2000.

10. Espinosa R, Gutiérrez MI, Mena-Muñoz H, Córdoba P. Domestic violence surveillance system: a model. Salud Publica Mex. 2008;50 supl 1:S12-8.

11. Guerrero R. ¿Qué sirve en la prevención de la violencia juvenil? Salud Publica Mex. 2008;50 supl 1:S86-92.

12. Franco Agudelo S. Violencia y salud en Colombia. Rev Panam Salud Publica. 1997; 1(2):93-103.

13. Rendón LF. Prioridades en salud de la Comuna 18 de Cali. Una aproximación hacia la orientación de los servicios de salud. Colomb Med. 2002;33:58-64.
14. Rendón LF, Bejarano M. Remisiones en el Valle del Cauca. Análisis y pautas para orientar la red de servicios. 2003-2004. Colomb Med. 2005;36:23-8.

15. CISALVA. Dimensionamiento de la violencia en Colombia. Documento de Trabajo R339. Washington, DC: Banco Interamericano de Desarrollo; 1998. Hallado en: http:// idbdocs.iadb.org/wsdocs/getdocument.aspx ?docnum=787964. Acceso el 14 de Junio de 2008.

16. Londoño JL, Guerrero R. Violencia en América Latina: epidemiología y costos. Capitulo 1. En: Londoño JL, Gaviria A, Guerrero R. Asalto al desarrollo. Violencia en América Latina. Washington, DC: Banco Interamericano de Desarrollo; 2000. Pp 11-58.

17. Concha-Eastman A, Espitia V, Espinosa R, Guerrero R. La epidemiología de los homicidios en Cali, 1993-1998: seis años de un modelo poblacional. Rev Panam Salud Publica. 2002;12(4):230-9.

18. Neme C. Diagnóstico municipal de la violencia y criminalidad en Jundiaí, Sao Paulo. Salud Publica Mex. 2008;50 supl 1:S19-28.

19. Orozco R, Borges G, Benjet C, Medina-Mora ME, López-Carrillo L. Traumatic life events and posttraumatic stress disorder among Mexican adolescents: results from a survey. Salud Publica Mex. 2008;50 supl 1:S29-37.

20. Bejarano M, Rendón LF, Rojas MC, Durán CA, Albornoz M. Caracterización de los pacientes con lesiones de causa externa mediante un sistema de vigilancia epidemiológica. Rev Colomb Cir. 2006;21(3):180-9.

21. Pohlman T, Bjerke HS, Offner P. Trauma scoring systems. E-medicine. Last updated: April 25, 2002. Hallado en http:/ / www.emedicine. $\mathrm{com} / \mathrm{med} /$ topic3214.htm. Acceso en Septiembre de 2005

22. Trauma,org [Sitio en Internet]. Revised trauma score. Hallado en: http://www. trauma.org/archive/scores/rts.html. Acceso el 9 de marzo de 2009.

23. Aprahamian C, Cattey R, Walter A, Gruchow $\mathrm{H}$, Seabrook G. Pediatric trauma score. Predictor of hospital resource use? Arch Surg. 1990;125(9):1128-31.

24. Pokorny W, Haller JA. Pediatric trauma. Chapter 44. En: Moore E, Mattox K, Feliciano D. Trauma. Second ed. Norwalk: Appleton \& Lange; 1991. Pp 689-702.

25. Buehler J. Surveillance. Chapter 22. En: Rothman K, Greenland S. Modern epidemiology. Second ed. Philadelphia: Lippincott Williams \& Wilkins; 1998. Pp 435-457.

26. UNICEF. Convención sobre los derechos del niño. Ginebra, 2002. Disponible en: http:// www2.ohchr.org/spanish/law/crc.htm Fecha de descarga: 09/03/2009.

27. Bejarano M, Rendón LF, Rojas M, Duran CA Albornoz M. Factores asociados con la intencionalidad en las lesiones de causa externa. Rev Colomb Cir 2006;21(4):215-24.

28. Borges G, Orozco R, Cremonte M, Buzi-Figlie $\mathrm{N}$, Cherpitel C, Poznyak V. Alcohol and violence in the emergency department: a regional report from the WHO collaborative study on alcohol and injuries. Salud Publica Mex. 2008;50.

29. Ávila-Burgos L, Medina-Solís CE, PérezNuñez R, Híjar-Medina M, Aracena-Genao B, Hidalgo-Solórzano E, Palma-Coca O. Prevalencia de accidents de tránsito no fatales en México: resultados de la ENSANUT 2006. Salud Publica Mex. 2008;50 supl 1:S38-47.

30. Arreola-Rissa C, Santos-Guzmán J, EsquivelGuzmán A, Mock CN. Traffic-related deaths in Nuevo Leon, Mexico: causes and associated factors. Salud Publica Mex. 2008;50 supl 1:S48-54.

31. Hidalgo-Solórzano E, Híjar M, Mora-Flores G, Treviño-Siller S, Inclán-Valadez C. Accidentes de tránsito de vehículos de motor en la población joven: evaluación de una intervención educativa en Cuernavaca, Morelos. Salud Publica Mex. 2008;50 supl 1:S60-8.

32. Espitia-Hardeman V, Vélez L, Muñoz E, Gutiérrez-Martínez MI, Espinosa-Vallín R, Concha-Eastman A. Efectos de las intervenciones diseñadas para prevenir las muertes de motociclistas en Cali, Colombia (1993-2001). Salud Publica Mex. 2008;50 supl 1:S69-77.

33. Mohan D. Traffic safety and city structure: lessons for the future. Salud Publica Mex. 2008;50 supl 1:S93-100.

34. Hernández N. Los jóvenes encuentran las sustancias cerca del colegio y de la U. Periódico El Tiempo, Bogotá Colombia. Hallado en: http://www.fungamma.org/observato rio/Consumoalcohol-psicoactivos-adolescen tesaprehendidos.htm. Acceso el 9 de marzo de 2009.

35. Rodríguez J, Muñoz E, Fandiño-Losada A, Gutiérrez MI. Evaluación de la estrategia de comunicación "Mejor hablemos" para promover la convivencia pacífica en Cali, 19962000. Rev Salud Publica. 2006;8(3):168-84.

36. Colimon KM. Fundamentos de epidemiología. Madrid: Ediciones Díaz de Santos; 1990.

Manuscrito recibido el 10 de octubre de 2007. Aceptado para publicación, tras revisión, el 3 de diciembre de 2008. 
ABSTRACT Objectives. To define and compare the types of injuries from external causes in patients more than and less than 18 years of age treated by the Mario Correa Rengifo Hospital.

Injuries from external causes in minors (less than 18 years of age) and adults at a hospital in Colombia

Methods. An analysis was conducted of data retrieved from the Sistema de Vigilancia de Lesiones de Causa Externa (Surveillance System for Injuries from External Causes) maintained by the Mario Correa Rengifo Hospital in Cali, Colombia, to compare minors (less than 18 years of age) with adults during January 2004-December 2007.

Results. A total of 4507 minors were seen, most of whom (69.3\%) were boys, with unintentional injuries (75.5\%); however, $88 \%$ of the intentional injuries were personto-person and $12 \%$ were self-inflicted. Injuries had occurred most frequently in the home $(44.1 \%)$ and on the street $(39.5 \%)$; the most frequent activity was recreation (53.3\%). The minors had more head injuries (odds ratio $[\mathrm{OR}]=1.62 ; P=0.0000$ ) and maxillofacial injuries $(\mathrm{OR}=1.49 ; P=0.0000)$ than did the adults. Alcohol had been consumed in $5.8 \%$ and drugs in $2.6 \%$, both of which increased intentional injuries (alcohol OR $=4.25$ and drugs OR $=1.56$ ).

Conclusions. The number of patients less than 18 years of age with injuries from external causes being treated at the Mario Correa Rengifo Hospital is constantly rising. The circumstances regarding injuries seen in children and adolescents differ from those of adults in that minors tend to suffer more unintentional injuries in the home or in the street where they play regularly. It is important that special educational and structural strategies be implemented to prevent injuries of this type.

Key words Violence, injuries, child health, adolescent health, Colombia. 